CASE REPORT

\author{
K. Koral \\ R.M. Kedzierski \\ B. Gimi \\ A. Gomez \\ N.K. Rollins
}

\title{
Subependymoma of the Cerebellopontine Angle and Prepontine Cistern in a 15-Year-Old Adolescent Boy
}

SUMmARY: A case of cerebellopontine angle and prepontine cistern subependymoma in a 15-year-old adolescent boy is presented with a review of the literature. Apparent diffusion coefficient values for subependymoma are reported. Differential considerations for the unusual location of this rare tumor are discussed.

S ubependymomas are rare intracranial neoplasms typically located in the subependymal regions of the ventricles. The patients usually present with slowly progressive symptoms, or the tumors are incidentally discovered at autopsy. ${ }^{1}$ The typical age of presentation is in the fifth or sixth decades. ${ }^{2}$ We report a rare case of subependymoma involving the cerebellopontine angle (CPA) and prepontine cistern in a 15-year-old adolescent boy and describe the subependymoma imaging characteristics, including apparent diffusion coefficient (ADC) values.

\section{Case Report}

A 15-year-old adolescent boy presented to the emergency department with a 4-year history of speech and balance difficulties. More recently, he developed progressive eye movement abnormalities. On examination, he had right partial cranial nerve (CN) VII and right CN VIII palsies, nystagmus, right-sided dysmetria, and hyperreflexia.

CT of the head showed a noncalcified, large posterior fossa mass resulting in hydrocephalus. MR imaging of the brain demonstrated a solid, exophytic lesion centered at the right CPA and extending anteriorly, obliterating the prepontine cistern. The mass measured $6.3 \times$ $5.7 \times 6.4 \mathrm{~cm}$ and showed T1 and T2 prolongation. The lesion did not arise or extend into the fourth ventricle, which was compressed and displaced posteriorly. Although there was marked displacement of the brain stem, particularly of pons and mesencephalon, no peritumoral edema was evident. The chronic nature of the lesion was underscored by scalloping of the dorsal surface of the clivus. There was moderate hydrocephalus without transependymal resorption of CSF, confirming chronicity of ventricular enlargement (Fig $1 A,-B$ ). On diffusionweighted imaging (DWI), the lesion did not show restricted diffusion. The ADC values were obtained using a circular region of interest with a diameter of $10 \mathrm{~mm}$ at 5 nonoverlapping locations in the tumor. The mean ADC value was $1.34 \times 10^{-3} \mathrm{~mm}^{2} / \mathrm{s}$ (range, $1.30-1.39 \times 10^{-3}$ $\left.\mathrm{mm}^{2} / \mathrm{s}, \pm \mathrm{SD} 0.04\right)$. The lesion demonstrated minimal punctate enhancement. There was no leptomeningeal tumor spread or spinal drop metastases.

The patient underwent a right-retrosigmoid and far-lateral craniotomy, and the bulk of the tumor was resected. Pathologic diagnosis

Received July 25, 2007; accepted after revision August 13.

From the Departments of Radiology (K.K., R.M.K., B.G., N.K.R.) and Pathology (A.G.), University of Texas Southwestern Medical Center at Dallas and Children's Medical Center, Dallas, Texas.

Please address correspondence to Korgun Koral, Children's Medical Center of Dallas, Department of Radiology, 1935 Motor St, Dallas, TX 75235; e-mail: korgun.koral@ utsouthwestern.edu

DOI 10.3174/ajnr.A0821 was subependymoma. The patient's symptoms improved gradually with residual CN palsies and speech difficulty. At 3-year follow-up, MR imaging of the brain did not show tumor regrowth.

\section{Discussion}

Subependymoma is a rare, low-grade (World Health Organization grade 1) glial neoplasm that is typically encountered in fifth and sixth decades of life. ${ }^{1-4}$ Because of its low-proliferative potential, it was suggested that subependymomas may represent a hamartoma rather than a neoplasm. ${ }^{3}$

Subependymomas commonly arise in the ventricular system. Infrequently, they are located in the septum pellucidum and spinal cord. ${ }^{5,6}$ The CPA and prepontine cistern involvement without extension into the fourth ventricle is uncharacteristic of subependymomas. Hoeffel et $\mathrm{al}^{4}$ described 4 cases with extension into CPA, but in all of them the tumors arose from the fourth ventricle. No CPA tumor was present in the 16 subependymomas reported by Ragel et $\mathrm{al}^{2}$ One CPA tumor was present among the 24 intracranial subependymomas reported by Chiechi et al. ${ }^{1}$

On CT, subependymomas are well-demarcated hyperattenuated lesions, with calcifications noted in up to $50 \%$ of the cases. ${ }^{1,6}$ On MR, subependymomas are generally isointense or hypointense on T1-weighted images and hyperintense on T2weighted images with respect to adjacent brain parenchyma. ${ }^{1,2,4}$ Usually there is no appreciable vasogenic edema of the adjacent parenchyma. Subependymomas show no enhancement or mild-to-moderate enhancement. ${ }^{1,2,4}$

To our knowledge, ADC values in a subependymoma were not published previously. In 2 reports, DWI of subependymomas was discussed, without reference to ADC values. Both lesions were in the fourth ventricle, and they were described as isointense/hyperintense on DWI. ${ }^{7,8}$ Rumboldt et $\mathrm{al}^{9}$ reported the use of $\mathrm{ADC}$ values in distinguishing pediatric cerebellar tumors. In this report, ADC values of 5 ependymomas were reported with a mean of $1.10 \pm 0.11 \times 10^{-3} \mathrm{~mm}^{2} / \mathrm{s}$. Our case has a mean tumor ADC value of $1.34 \pm 0.11 \times 10^{-3} \mathrm{~mm}^{2} / \mathrm{s}$, which is slightly higher than those of ependymoma, but not as high as those of juvenile pilocytic astrocytoma (JPA). ${ }^{9}$

The differential diagnosis of a large CPA mass in a young person includes ependymoma, JPA, medulloblastoma, atypical teratoid/rhabdoid tumor, brain stem glioma, choroid plexus tumors, hemangioblastoma, arachnoid cyst, and epidermoid. A rare exophytic low-grade brain stem glioma may potentially be 

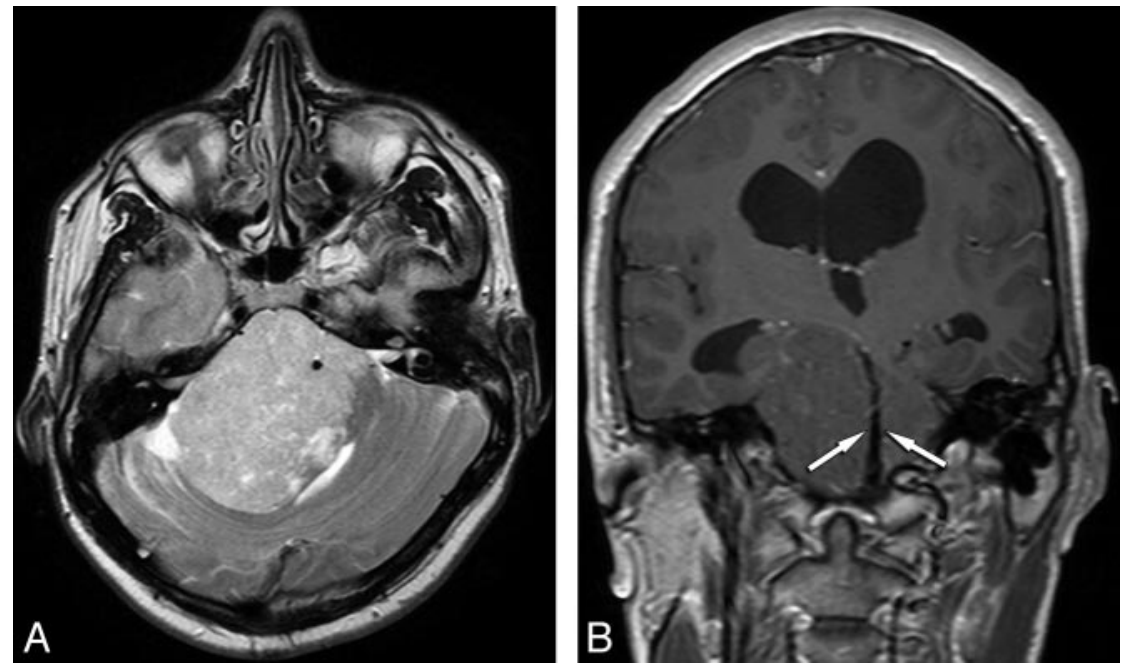

Fig 1. A, Axial T2-weighted image shows a large, solid hyperintense mass in the right CPA and prepontine cistern, displacing the fourth ventricle to left. The signal intensity void of basilar artery is maintained but is entirely circumscribed by tumor. B, Coronal contrast-enhanced T1-weighted image shows the patent basilar artery (arrows) and obstructive hydrocephalus. On the left, the tumor traverses the tentorial incisura and displaces the third ventricle to the left. There is minimal punctate enhancement. difficult to distinguish from a case of subependymoma similar to ours. Subependymoma is a very rare pediatric tumor, but lack of vasogenic edema in subependymoma may be a distinguishing feature from the otherwise similar ependymoma.

\section{References}

1. Chiechi MV, Smirniotopoulos JG, Jones RV. Intracranial subependymomas: CT and MR imaging features in 24 Cases. AJR Am J Roentgen 1995; 165:1245-50

2. Ragel BT, Osborn AG, Whang K, et al. Subependymomas: an analysis of clinical and imaging features. Neurosurgery 2006;58:881-90

3. Prayson RA, Suh JH. Subependymomas: clinicopathological study of 14 tumors, including comparative MIB-1 immunohistochemical analysis with other ependymal neoplasms. Arch Pathol Lab Med 1999;123:306-09
4. Hoeffel C, Boukobza, Polivka M, et al. MR manifestations of subependymomas. AJNR Am J Neuroradiol 1995;16:2121-29

5. Rath TJ, Sundgren PC, Brahma B, et al. Massive symptomatic subependymoma of the lateral ventricles: case report and review of the literature. Neuroradiology 2005;47:183-88

6. Jooma R, Torrens MJ, Bradshaw J, et al. Subependymomas of the fourth ventricle: surgical treatment in 12 cases. J Neurosurg 1985;62:508-12

7. Fontenele GI, Okamoto K, Ito J, et al. Symptomatic child case of subependymoma in the fourth ventricle without hydrocephalus. Radiat Med 2001; 19:37-42

8. Quadery FA, Okamoto K. Diffusion weighted MRI of haemangioblastomas and other cerebellar tumours. Neuroradiology 2003;45:212-19

9. Rumboldt Z, Camacho DL, Lake D, et al. Apparent diffusion coefficient for differentiation of cerebellar tumors in children. AJNR Am J Neuroradiol 2006; 27:1362-69 